\title{
Acute Lymphocytic Leukemia Detection and Classification (ALLDC) System
}

\author{
Jamila Harbi, PhD \\ Computer Science Dept. \\ College of Science \\ Al- Mustansiriyah University \\ Baghdad, Iraq
}

\author{
Rana Ali \\ Computer Science Dept. \\ College of Science \\ Al- Mustansiriyah University \\ Baghdad, Iraq.
}

\begin{abstract}
In order to improve patient diagnosis various image processing software are developed to extract useful information from medical images. An essential part of the diagnosis and treatment of leukemia is the visual examination of the patient's peripheral blood smear under the microscope. Morphological changes in the white blood cells are commonly used to determine the nature of the malignant cells, namely blasts. Morphological analysis of blood slides are influenced by factors such as hematologists experience and tiredness, resulting in non standardized reports. So there is always a need for a cost effective and robust automated system for leukemia screening which can greatly improve the output without being influenced by operator fatigue. This paper presents an application of image segmentation, feature extraction, selection and cell classification to the recognition and differentiation of normal cell from the blast cell. The system is applied for 108 images available in public image dataset for the study of leukemia. The methodology demonstrates that the application of pattern recognition is a powerful tool for the differentiation of normal cell and blast cell leading to the improvement in the early effective treatment for leukemia.[1]
\end{abstract}

\section{Keywords}

AML, ALL, ALLDC, ALLCD

\section{INTRODUCTION}

Medical diagnosis is the procedure of identifying a disease by critical analysis of its symptoms and is often aided by a series of laboratory tests of varying complexity. Accurate medical diagnosis is essential in order to provide the most effective treatment option [2].

Leukemia, a blood cancer, is one of the commonest malignancies affecting both adults and children. It is a disease in which digital image processing and machine learning techniques can play a prominent role in its diagnostic process [3].

Leukemia is classified as either acute or chronic based on the rapidity of the disease progression. Acute leukemia can be further classified to acute lymphoblastic leukemia (ALL) and acute myeloid leukemia (AML) based on the cell lineage. The treatment protocol is allocated based on the leukemia type. Fortunately, leukemia like many other cancer types are curable and patient survival and treatment can be improved, subject to accurate diagnosis [3]. In particular, this thesis focuses on Acute Lymphoblastic Leukemia (ALL), with the main objective to develop a methodology to detect and classify Acute Leukemia blast cells based on image processing techniques using peripheral blood smear images [4].
The methodology presented in this thesis consisted of several stages namely, image segmentation, feature extraction and, classification.

\subsection{Typical Blood Microscope Image}

A typical blood microscope image is plotted in Figure (1) the principal cells present in the peripheral blood are red blood cells (RBCs), and the white blood cells (WBCs, leucocytes). Granules are contained in Leucocyte cells called granulocytes (collected by neutrophil, basophil, and eosinophil). The cells which are without granules are called granulocytes (composed of monocyte and lymphocyte) [5].

Conventionally, manual counting done under the microscope to count the white blood cells in leukemia slides. This way is bothersome and time consuming if the process of counting is interrupted, it must be started all over again. Therefore, manual counting prone have errors in procedure and put an insupportable amount of pressure on the medical laboratory technicians. Though there are many hardware solutions such as the Automated Hematology Counter to perform counting, certain developing countries are not capable to set up such expensive machines in all hospital laboratories in all over country [6].

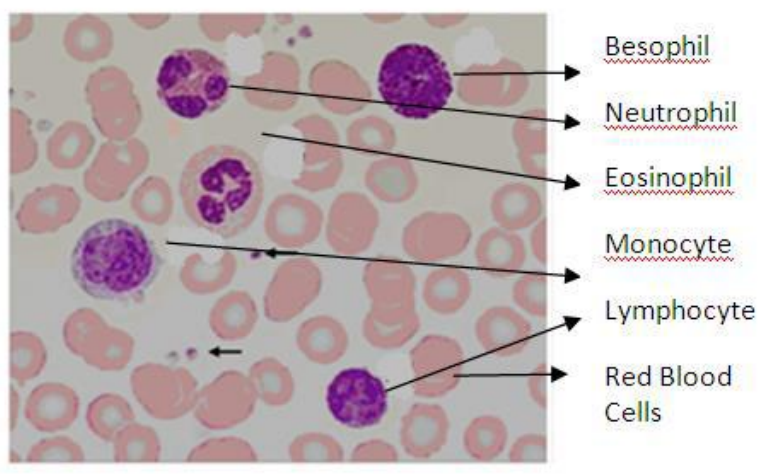

Fig (1): Typical blood microscope image Proposed Acute LymphocyticLeukemia Detection and Classification

(ALLDC) System

Medical Image in recent is very important side of image processing technique especially in image detection and classification cancer diseases such as Acute LymphocyticLeukemia. Therefore many of the image processing systems for leukemia detection in the literature are still at prototype stage and have proposed techniques to refine the segmentation or to refine membrane or to detect incorrect segmentations of white cells. Also most of systems developed work on sub-images where only one nucleus per image is presented under the field of view. 
Our goal is to overcome these techniques and also increase the accuracy of the classifier system. To achieve this goal, theproposed Acute LymphocyticLeukemia Detection and Classification (ALLDC) System will be has follows four major processing steps such as: image preprocessing, segmentation, feature extraction and classification steps.

ALLDC system has two stages of blood image segmentation and feature extraction after preprocessing done some operations as shown in Figure (2).

ALLCD System steps will be explained in more details:

\section{Image Segmentation}

Medical image segmentation becomes vital process for its proper detection and diagnosis of diseases. WBCs segmentation becomes important issue because differential between them such as normal or leukemia diseased. Otsu's method is a technique frequently applied to image segmentation. Its basic objective is to classify the pixels of a given image into two classes: those pertaining to an object and those pertaining to the background. The proposed segmentation steps can be summarized in Fig (2):

\section{Image Preprocessing}

The main aim of image preprocessing is to enhancethe visual appearance of images. In this step, the JPEG image is converted into BMP RGB-24 bit color space
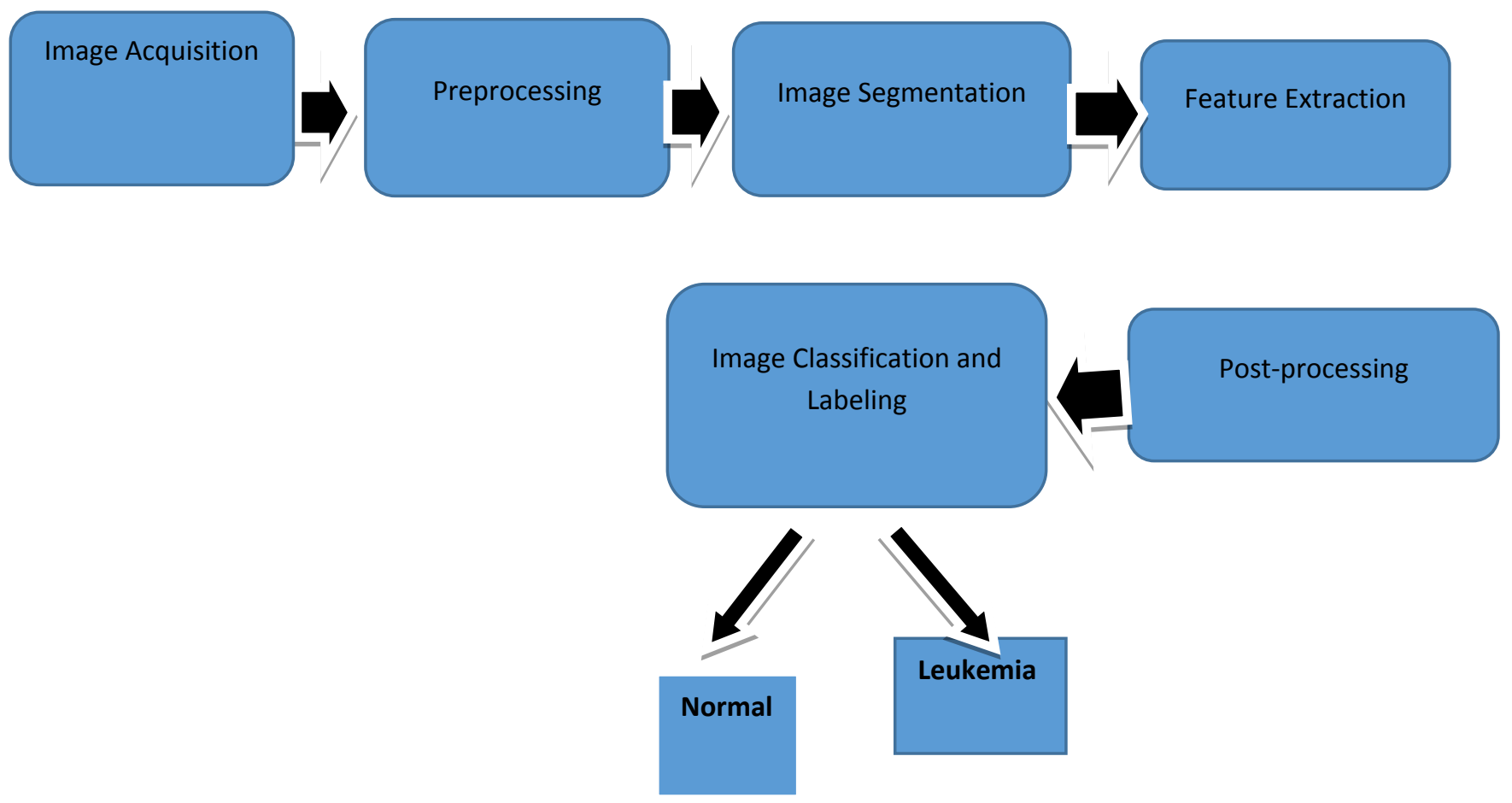


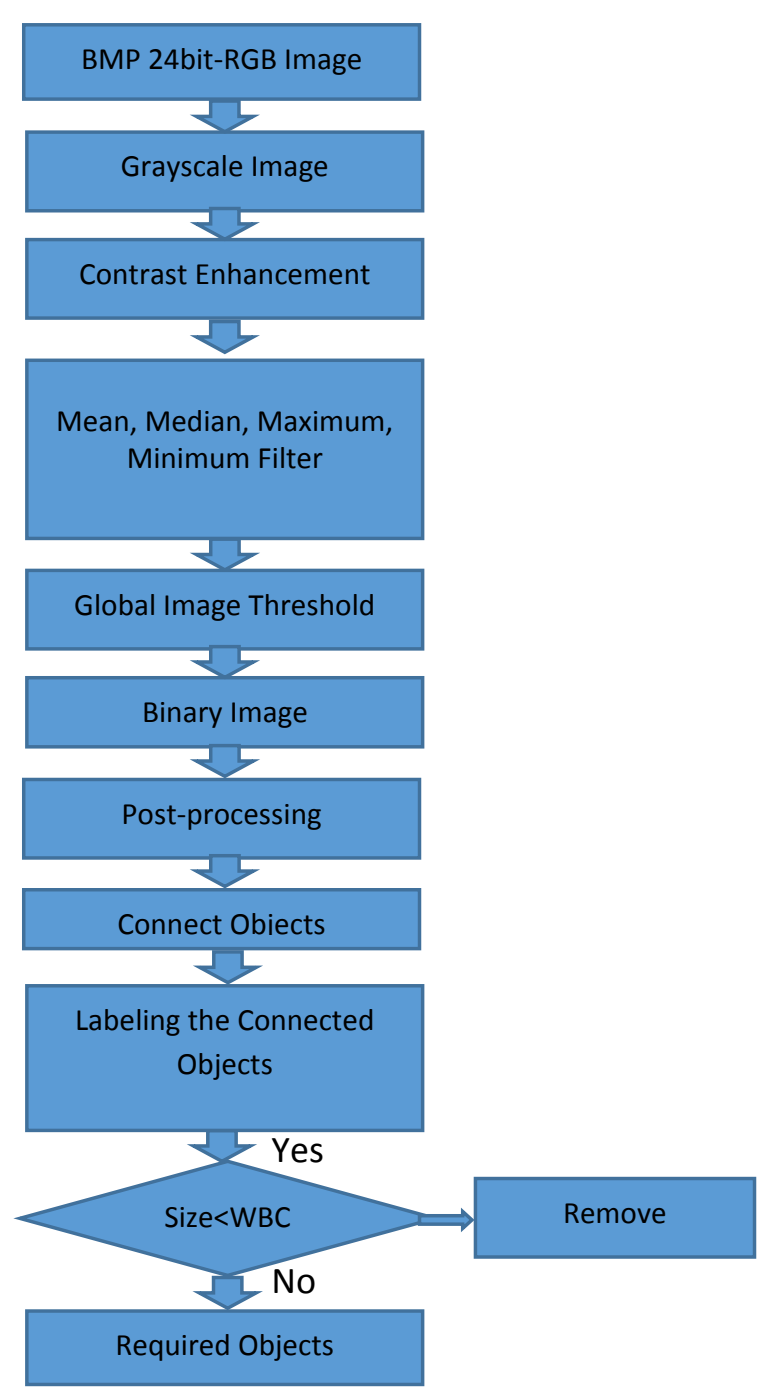

Fig (3): The proposed steps to segment WBCs

All steps of segmentation processes shown in Figure (3) will explain in more details:

\section{Image Conversion (RGB to Grayscale)}

All grayscale algorithms utilize the same basic three-step processes:

1. Get the red, green, and blue values of a pixel with 24 bit

2. Use easy math to turn those value into a single gray value with 8 bit

3. Replace the original red, green, and blue values with the new gray value

When describing grayscale algorithms, we going to focus on step 2 - using math to turn color values into a grayscale value. For each image pixel with red, green and blue values of $(\mathrm{R}, \mathrm{G}$, B) we use eq. (2-1) of the luminosity conversion method.

\section{Contrast Enhancement}

Histogram Equalization and Linear Contrast Starching as well as the addition and subtraction process will be used to produce enhancement images.

\section{A. Histogram Equalization}

Histogram Equalization illustrated in algorithm (1) when applied on grayscale image will produced an equalize brightness gray levels of the image.

Algorithm (1): Histogram Equalization

Input: grayscale 8-bit image $\{x\}$.

Output: equalized image $\{\mathrm{y}\}$ will produced.

1. Image size calculated $n=$ row pixels $x$ column pixels

2. Compute the image's histogram for each pixel value i:

$\mathrm{H}_{\mathrm{x}}(\mathrm{i})=\frac{\mathrm{n}_{\mathrm{i}}}{\mathrm{n}}, 0 \leq \mathrm{i}<L \quad$ (Where $\mathrm{L}$ total number of gray levels $2^{8}=256$ )

3. Compute the cumulative distribution function corresponding to $\mathrm{H}_{\mathrm{x}}$ as in formula below:

$$
\operatorname{cdf}_{x}(i)=\sum_{j=0}^{i} H_{x}(j) \ldots . .(?)
$$

4. Calculate the minimum $\mathrm{CDF}$ value $\left(C D F_{\min }\right)$

5. Apply the equalization function for each pixel $i$ in $\{x\}$

$$
\mathrm{i}_{\text {new }}=\operatorname{round}\left(\frac{\mathrm{CDF}(\mathrm{i})-\mathrm{CDF}_{\min }}{\mathrm{n} \times \mathrm{L}}\right) \ldots \ldots(?)
$$

\section{A. Linear Contrast Starching}

Linear Contrast Starching illustrated in algorithm (2) when applied on grayscale image will produce enhanced image.

Algorithm (2): Linear Contrast Stretching Histogram

Input: Grayscale 8-bit image $\{\mathrm{x}\}$

Output: Enhanced image $\{\mathrm{y}\}$ will produced.

1. Calculate $\mathrm{L}=28-1(\mathrm{~L}=255)$

2. Calculate the minimum intensity value a.

3. Calculate the maximum intensity value $b$.

4. Calculate the difference between the minimum and maximum values $d=|b-a|$

5. Apply the linear stretching function for each pixel (i) in an image $\{x\}$

$$
i_{\text {new }}=\left[\frac{(i-a)}{d}\right] \times L \ldots .(3-3)
$$

\section{A. Combine Process}

In this step, the combine process will brighten most of the details in the enhanced image except the nuclei since by performing the new image called addition image.

$$
\mathrm{I}_{1}=\mathrm{A}+\mathrm{B}
$$

\section{B. Subtraction Process}

The subtraction process between the enhanced images resulted from histogram equalization and histogram linear contrast stretching. In this step, we notice the subtraction process will highlight all the objects and its borders in the image including the cell nuclei.

$$
\mathrm{I}_{2}=\mathrm{A}-\mathrm{B}
$$




\section{Further Enhancement Process}

Two enhanced image resulted from combine and subtraction process will be summed to get new image contain only the WBCs. $I_{3}$ is the new image of enhancement process, i.e.

$$
\mathrm{I}_{3}=\mathrm{I}_{1}+\mathrm{I}_{2} \ldots \text { (3) }
$$

In this step, sum process will remove almost all the other blood components while retaining the nuclei with minimum effect of distortion on the nuclei part of the white blood cells.

\section{Minimum Filter}

Noise may be occurred when applying the enhancement process on the image therefore this image need to use noise removal filter. In ALLCD proposed system minimum filter is applied with size $3 \times 3$ of window on whole image $\mathrm{I}_{3}$. The filter will scanned each pixel in image $\mathrm{I}_{3}$ first by row and then second by column. The filter works by applying the window $3 \times 3$ pixels then sorted it ascending, changing the pixel intensity for each pixel in the $3 \times 3$ window with the first pixel intensity (minimum intensity).

\section{Global Image Threshold}

The filtered image which is resulted from previous section III must be converted from grayscale to binary image. Thresholding technique (Otsu's method) is used to make this conversion. The steps of applying Otsu's method shown in algorithm (3) and the binary image $\mathrm{I}_{3}$ produced.

Input: Grayscale image $\{x\}$

Output: Binary image

1. Calculate image histogram:

$$
\mathrm{H}_{\mathrm{x}}(\mathrm{i})=\frac{\mathrm{n}_{\mathrm{i}}}{\mathrm{n}}, \quad 0 \leq \mathrm{i}<L
$$

(Where L total number of gray levels $2^{8}=256$ )

2. Calculate cumulative histogram:

$$
\mathrm{C}=\sum_{\mathrm{i}=0}^{\mathrm{L}} \mathrm{H}(\mathrm{i}) * \mathrm{i}
$$

3. Select a threshold and referred as $\mathrm{T}$,

3.1. Initialize weight background $\mathrm{W}_{\mathrm{b}}=0$ and foreground $\mathrm{W}_{\mathrm{f}}=0$, cumulative histogram sum $\mathrm{b}=0$, between $=0, \quad$ threshold $=0$, MAX $=0$

3.2. Initial intensity $\mathrm{i}=0$

3.3. Calculate $\mathrm{w}_{\mathrm{b}}=\mathrm{w}_{\mathrm{b}} * \mathrm{H}(\mathrm{i})$

3.4. If $\mathrm{w}_{\mathrm{b}}=0$ get next intensity, GOTO 3.3

3.5. Calculate $\mathrm{W}_{\mathrm{f}}=\mathrm{n}-\mathrm{W}_{\mathrm{b}}$

3.10. If $\mathrm{W}_{\mathrm{f}}=0$ set intensity $\mathrm{i}=255$ GOTO 3.2

3.11. Calculate $\operatorname{sum}_{\mathrm{b}}=\sum_{\mathrm{i}=0}^{\mathrm{L}} \mathrm{H}(\mathrm{i}) * \mathrm{i}$

3.12. Calculate mean value for background \& foreground

$\mathrm{m}_{\mathrm{b}}=\frac{\text { sumb }}{\mathrm{w}_{\mathrm{b}}}$, and $\mathrm{m}_{\mathrm{f}}=\frac{\mathrm{C}-\text { sumb }}{\mathrm{w}_{\mathrm{f}}}$

3.13. Calculate between within class variance

Between $=\mathrm{w}_{\mathrm{b}} * \mathrm{w}_{\mathrm{f}} *\left(\mathrm{~m}_{\mathrm{b}}-\mathrm{m}_{\mathrm{f}}\right) *\left(\mathrm{~m}_{\mathrm{b}}-\mathrm{m}_{\mathrm{f}}\right)$

3.14 IF between $<=$ MAX then increment i, GOTO 3.3.

3.15 . MAX=between

3.16 Threshold $=\mathrm{i}$

3.17 IF i=255 GOTO 3.15

3.18 GOTO 3.3
3.19 Final global threshold, $\mathrm{T}=$ threshold

4. Binaries Image $=$ gray scale image $>\mathrm{T}$

5. Thus the transformation of an input image $\mathrm{X}$ into a binary image $\mathrm{B}$ at a selected threshold $\mathrm{T}$, can be represented as follows:

(a) bij $=1$ IF $\quad x_{i j}>T$

(b) $\quad$ bij=0 IF $X_{\mathrm{ij}}<\mathrm{T}$

Here $b_{i j}=1$, for the object or foreground pixels and $b_{i j}=0$, for the background pixels.

\section{Morphological Operations}

Morphological opening is used to remove the small groups of pixels which can form false objects. Morphological opening is done by applying erosion followed by dilation. Erosion is applied with a disk structuring element $7 \times 7$ shape shown in figure (4).

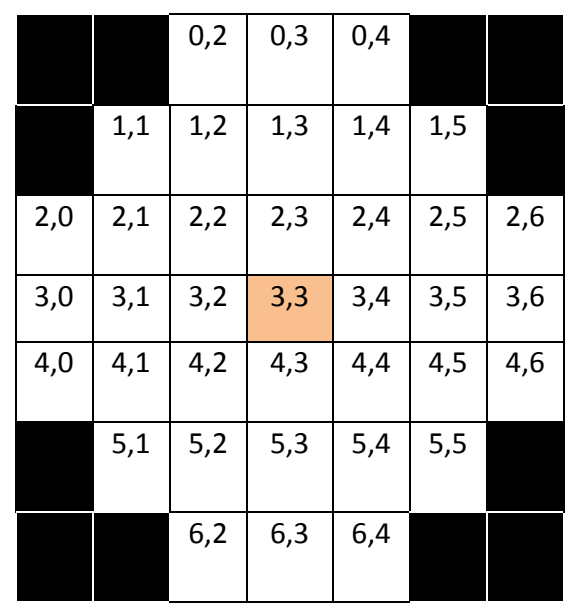

Fig (4): Disk Structure Element 7x70pening morphological operation illustrated in algorithm (4) which produce a new opening image $B$ generated image.

\section{Connected Objects}

Connected object algorithm will be used in our proposal system. 8-connectivity is used to count and locate the nuclei of the WBCs. In this process the connectivity checks are carried out by checking neighbor pixels' labels of the opening image resulted from the previous process.

Once the initial labeling and equivalence recording is completed, the second pass merely replaces each pixel label with its equivalent disjoint-set representative element.

A faster-scanning algorithm for connected-region extraction is presented below.

\section{a. The First Pass}

1. Iterate through each element of the data by column, then by row (Raster Scanning)

2. If the element is not the background

i. Get the neighboring elements of the current element

ii. If there are no neighbors, uniquely label the current element and continue

iii. Otherwise, find the neighbor with the smallest label and assign it to the current element

iv. Store the equivalence between neighboring labels 


\section{b. The Second Pass}

1. Iterate through each element of the data by column, then by row.

Algorithm (4): Opening morphological operation

Input: binary image B.

Output: New opening image B generated

1. Disk structure element $7 \times 7$ is applied.

2. Erosion process apply on B-image

2.1 Scan B-image row by row and then column by column

2.2 Compare the $\mathrm{SE}_{7 \times 7}$ elements with the corresponding elements in the $\mathrm{B}$-image.

2.3 IF no complete matching, the core element $\mathrm{b}_{3 \times 3}=0$ GOTO 3.5

$2.4 \mathrm{~B}_{3 \times 3}=1$

2.5 IF the whole rows and columns are covered with overlap 6 in both, GOTO 3.7

2.6 Repeat 3.1 to 3.5

2.7 New eroded B- image is produced.

3. Dilation process applies on eroded B- image generated from step 3 .

3.1 Scan B-image row by row then column by column

3.2 Compare the $\mathrm{SE}_{7 \times 7}$ elements with the corresponding elements in the B-image.

3.3 IF one element matching, the core element $\mathrm{b}_{3 \times 3}=255$ GOTO 4.5

$3.4 \quad \mathrm{~B}_{3 \times 3}=1$

3.5 IF the whole rows and columns are covered with overlap 6 in both, GOTO 4.7

3.6 Repeat 3.1 to 3.5

3.7 New dilation B-image is produced.

2. If the element is not the background re-label the element with the lowest equivalent label. Here, the background is a classification, specific to the data, used to distinguish salient elements from the foreground. If the background variable is omitted, then the two-pass algorithm will treat the background as another region. For example,

i. The array from which connected regions are to be extracted is given below (8-connectivity based). First different binary values are assigning to elements in the graph. Attention should be paid that the " $0 \sim 1 "$ values written on the center of the elements in the following graph are elements' values. While, the " $1,2, \ldots, 7 "$ values in the next two graphs are the elements' labels. The two concepts should not be confused.

ii. After the first pass, the following labels are generated. A total of 7 labels are generated in accordance with the conditions highlighted above.
Table (1) label relationships

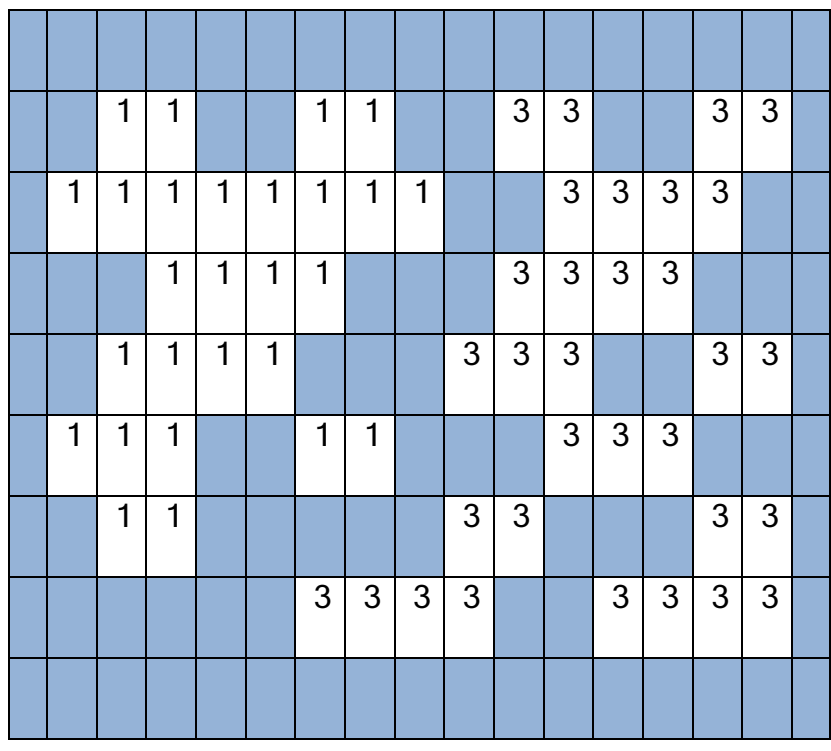

The label equivalence relationships generated are in Table (1)

Table (2) label equivalence relationships

\begin{tabular}{|c|c|}
\hline Set ID & $\begin{array}{l}\text { Equivalent } \\
\text { Labels }\end{array}$ \\
\hline 1 & 1,2 \\
\hline 2 & 1,2 \\
\hline 3 & $3,4,5,6,7$ \\
\hline 4 & $3,4,5,6,7$ \\
\hline 5 & $3,4,5,6,7$ \\
\hline 6 & $3,4,5,6,7$ \\
\hline 7 & $3,4,5,6,7$ \\
\hline
\end{tabular}

i. Array generated after the merging of labels is carried out. Here, the label value that was the smallest for a given region "floods" throughout the connected region and gives two distinct labels, and hence two distinct labels.

ii. Final result in color to clearly see two different regions that have been found in the array.

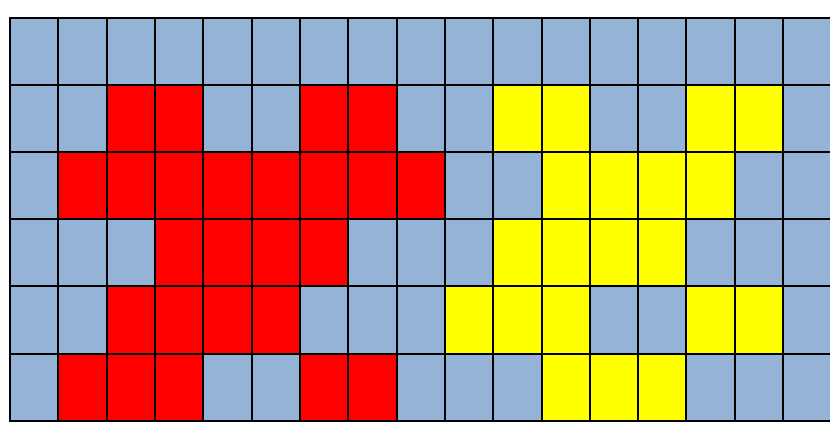




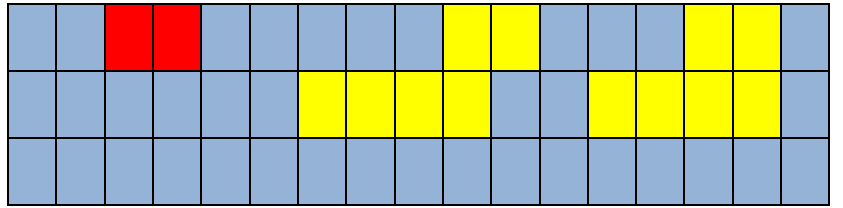

\section{Feature Extraction}

Feature extraction means to transfer the input data into different set of features. In ALLCD proposal system three features of lymphocyte cells have been observed, area, perimeter and circularity because the shape of the nucleus is important feature for differentiation of blasts.

\section{K-Nearest Neighbors Classification Algorithm (KNN)}

The final stage of ALLCD proposed system give a decision of the WBCs are normal or leukemia diseased by using the $\mathrm{K}$ Nearest Neighbors Classification Algorithm (KNN). All steps of applying KNN algorithm shown as follow:

1. Choose a value for the parameter $\mathrm{k}$ (the proposed system $\mathrm{k}=1$ )

2. Input: Give a sample of $\mathrm{N}$ examples and their classes.

3. The class of an sample $\mathrm{x}$ is $\mathrm{c}(\mathrm{x})$ :

4. Give a new sample y:

5. Determine the k-nearest neighbors of $y$ by calculating the distances.

6. Combine classes of these y examples in one class $\mathrm{c}$

7. Output : The class of $y$ is $c(Y)=c$

\section{Feature Extraction}

Feature extraction means to transfer the input data into different set of features. In ALLCD proposal system three features of lymphocyte cells have been observed, area, perimeter and circularity because the shape of the nucleus is important feature for differentiation of blasts.

\section{K-Nearest Neighbors Classification Algorithm (KNN)}

The final stage of ALLCD proposed system give a decision of the WBCs are normal or leukemia diseased by using the $\mathrm{K}$ Nearest Neighbors Classification Algorithm (KNN). All steps of applying $\mathrm{KNN}$ algorithm shown as follow:

8. Choose a value for the parameter $\mathrm{k}$ (the proposed system $\mathrm{k}=1$ )

9. Input: Give a sample of $\mathrm{N}$ examples and their classes.

10. The class of an sample $\mathrm{x}$ is $\mathrm{c}(\mathrm{x})$ :

11. Give a new sample y:

12. Determine the k-nearest neighbors of $y$ by calculating the distances.

13. Combine classes of these y examples in one class $\mathrm{c}$

14. Output: The class of $\mathrm{y}$ is $\mathrm{c}(\mathrm{Y})=\mathrm{c}$

\section{RESULTS AND DISCUSSION}

The original smeared image converted to a grayscale image, which presents the nuclei of the WBCs the darkest areas in the image as shown in the Figure (5). The grayscale image resulted from conversion process, the all components of gray levels concentered in the middle of dynamic range in other words the resulted grayscale image is low contrast in intensity therefore this image needs to enhanced the intensity components. Figure (6) shown the histogram of converted grayscale image

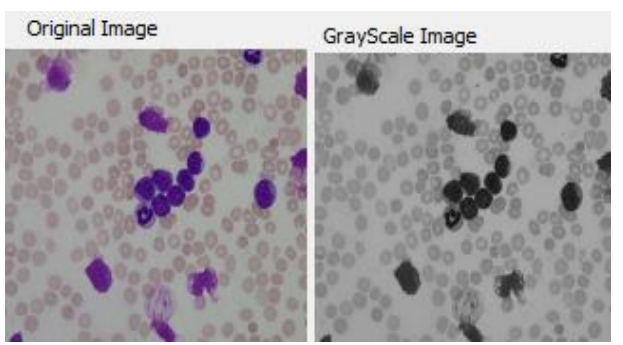

Fig (5): Grayscale after Conversion Process

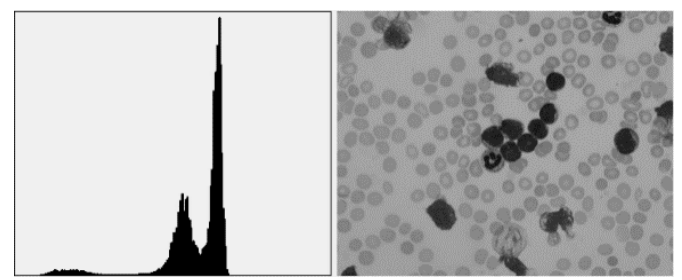

Fig (6): Histogram of Converted Grayscale Image

The histogram of gray scale image in Figure (6) illustrated this image is low contrast, all components of brightness level concerted in the middle range of gray level distribution therefore this image need to enhanced.

Histogram equalization and linear contrast starching as well as the addition and subtraction process will be used to produce enhancement images. Figure (7) shown the histogram equalization image (the enhanced A-image). The histogram equalization image is shown in Figure (8).

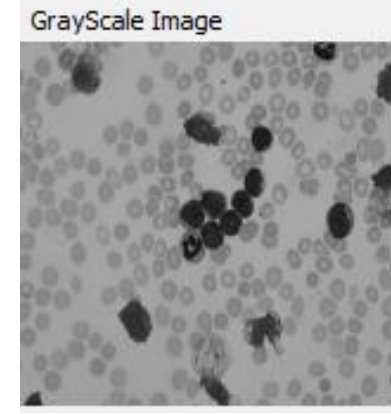

(a) Histogram equalized image-A

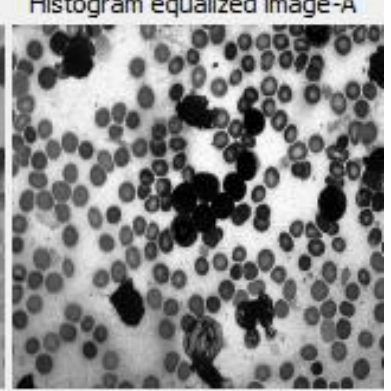

(b)
Fig (7): Histogram equalization of grayscale image:

a) Grayscale image, b) equalized image.

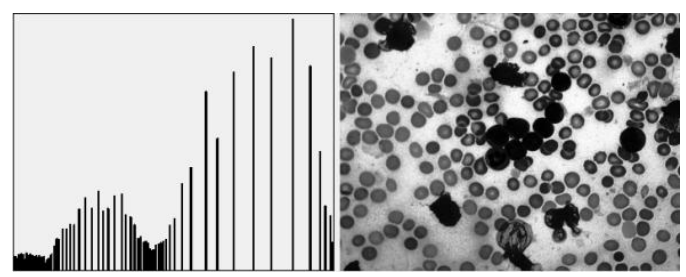

Fig (8): Histogram equalized image and

histogram equalized image histogram

Linear Contrast Starching illustrated enhanced B-image shown in Figure (9). Figure (10) shown how the image is enhanced the intensity by distributing the gray values in most dynamic range of gray levels. Also we can compared with the 
enhancement by using equalization histogram which is shown in figure (8), it's obviously equalization is best than linear contrast stretching because the gray values distributed in all the dynamic range but in linear contrast stretching histogram the gray values occupied the most dynamic range.

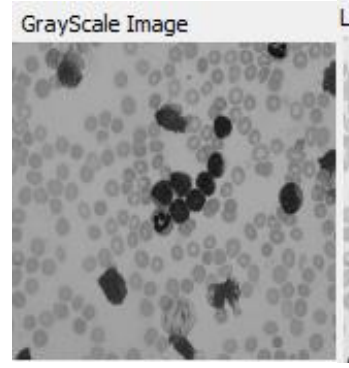

(a)

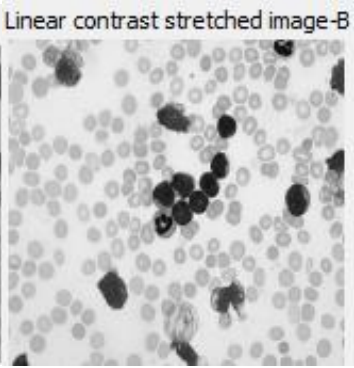

(b)
Fig (9): linear contrast starching of grayscale image:

a) Grayscale image, b) enhanced image

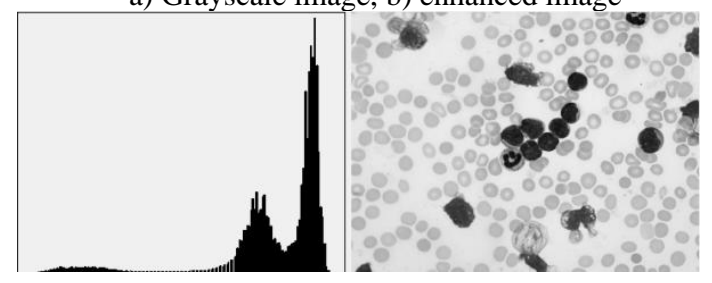

Fig (10): linear contrast starching grayscale image and linear contrast histogram

Let us called the resulted image from the process of histogram equalization A-Image and B-Image resulted from histogram linear contrast stretching, now combine A-image and B-image with addition process $(\mathrm{A}+\mathrm{B})$ as shown in figure (11), the compound image called $\mathrm{I}_{l}$, all the resultant pixel values exceeding the intensity value of 225 is replaced with 255 . From figure (11) we noticed that only the pixel values of white cell of blood i.e., the low intensity value can obtain but they occupied all the dynamic range.

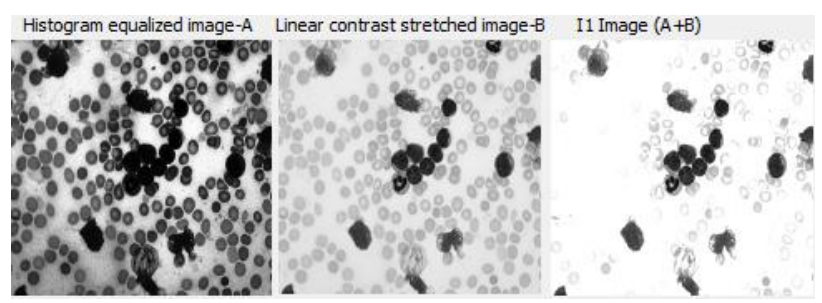

Fig (11): Combine Process: Addition
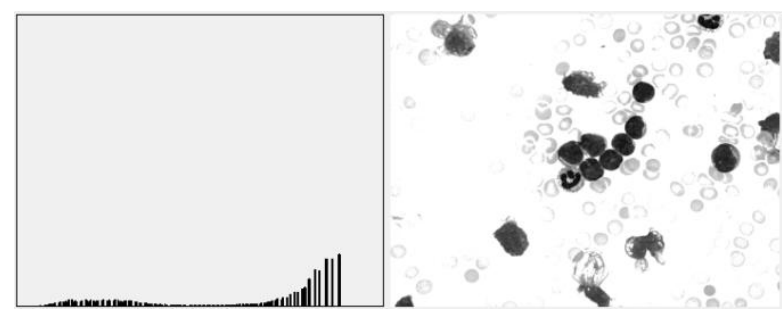

Fig (12): Addition $I_{1}$ image with its histogram

Combine A-image and B-image with subtraction process (BA) as shown in figure (13). Also, this enhancement obviously in figure (14), we can see all components of histogram towards to increase the intensity and enhanced the brightness. Suppose the subtraction image calledI ${ }_{2}$.

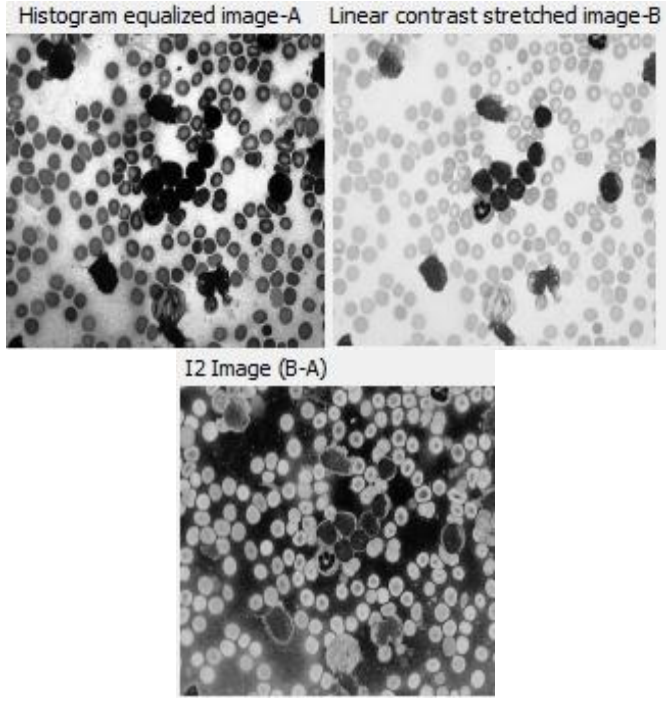

Fig (13): Subtraction Process

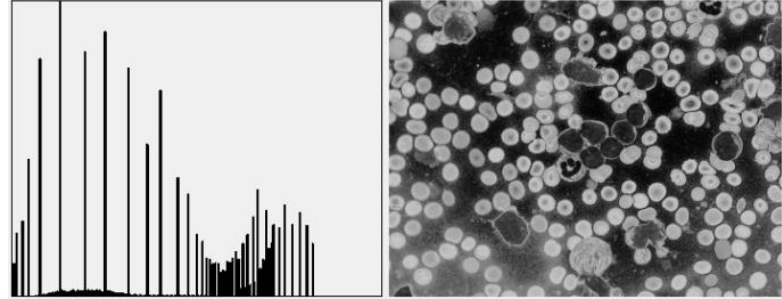

Fig (14): Subtraction Process $I_{2}$-image with its histogram

Two enhanced image resulted from addition $I_{l}$ and subtraction processes $I_{2}$ will be summed to get new image contain only the WBCs. Suppose $I_{3}$ is the new image name of enhancement process.

In this step, sum process will remove almost all the other blood components while retaining the nuclei with minimum effect of distortion on the nuclei part of the white blood cells. Figure (15) is shown the resulted $\mathrm{I}_{3}$-image after enhancing with I3-histogram.
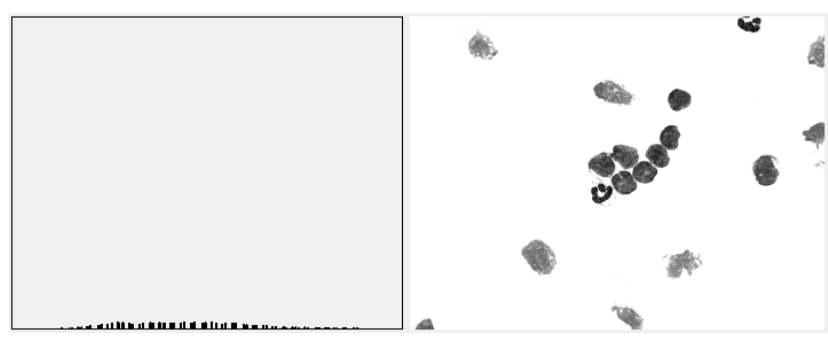

Fig (15): Further Processes after $I_{3}$-image enhancing with its histogram

After minimum filter is produced $\mathrm{I}_{3}$-image which can be seen in figure (16), whereas figure (17) shown its histogram after minimum filter is done. 


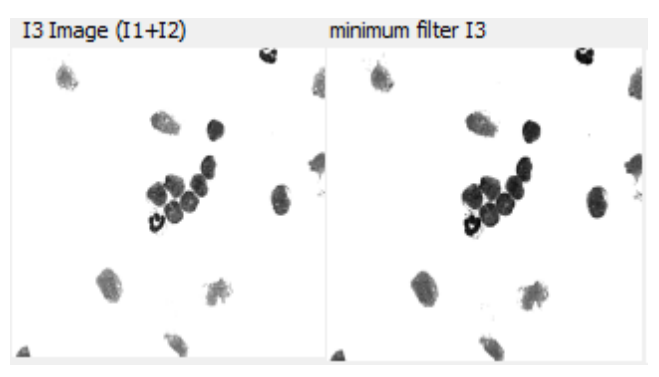

Fig (16): Image after applying minimum Filter

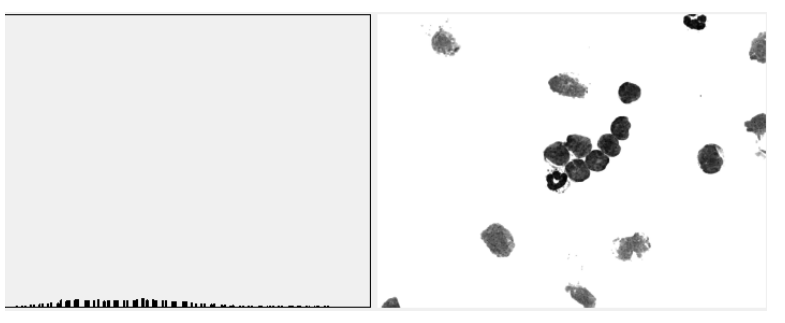

Fig (17): Image after minimum Filter is applied with its histogram

Minimum filter $\mathrm{I}_{3}$-image must be converted from gray scale to binary image. Thresholding technique (Otsu's method) is used to make this conversion to produce the binary image as in figure (18).

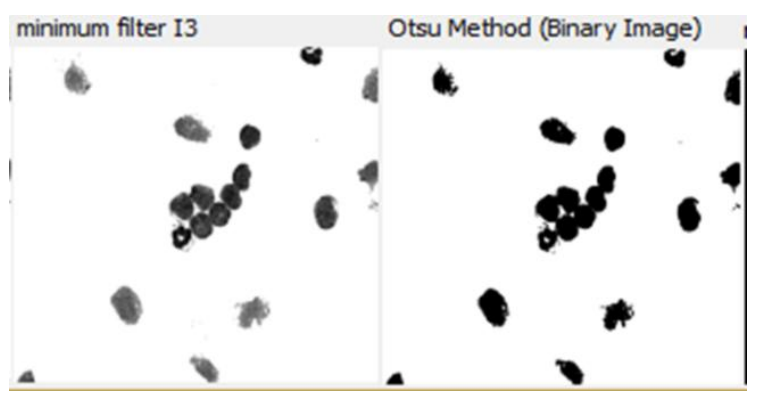

Fig (18): Converting grayscale image to binary image
Morphological opening operations when applies on the binary image produced the image as shown in the figure (19):

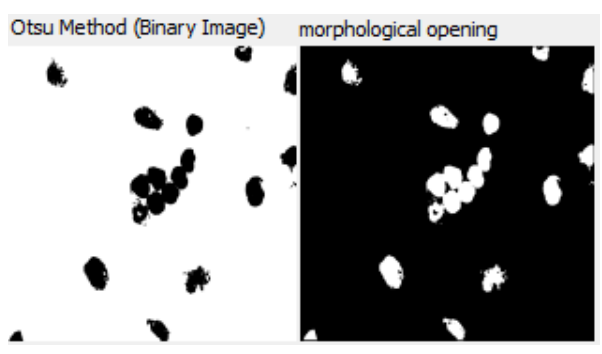

Fig (19): Morphological opening on binary image

The connectivity process checks are carried out by checking neighbor pixels labels of the opening image resulted from the previous process and the connected objects are shown in figure (20).

From connected objects step we will check the relative size (area) of each object with respect to average WBC area all the tests are shown as in table (3). The 50\% value is used as a minimum nucleus segment threshold. This value was chosen by trials which gave the best accuracy of segmentation. Table (4) is shown the required objects of segmentation processes as in figure (21)

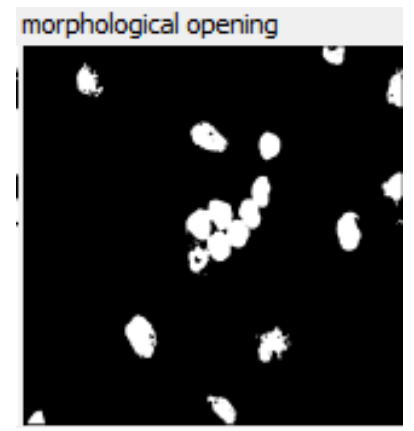

Fig (20): Morphological opening on binary image

\begin{tabular}{|c|c|c|c|c|c|c|c|c|c|c|c|}
\hline $\begin{array}{l}\text { WBC } \\
\text { nuclei } \\
\#\end{array}$ & size & $\begin{array}{l}\mathbb{0} \\
\mathbb{\pi} \\
\stackrel{\pi}{0} \\
\mathbb{\pi}_{0}\end{array}$ & $\begin{array}{l}\text { WBC } \\
\text { nucle } \\
\text { i \# }\end{array}$ & Size & $\begin{array}{l}\stackrel{0}{\mathbb{\pi}} \\
\underset{\pi}{0} \\
\sum_{\pi}^{\pi}\end{array}$ & $\begin{array}{l}\text { WBC } \\
\text { nucle } \\
\text { i \# }\end{array}$ & size & 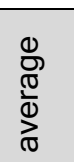 & $\begin{array}{l}\text { WBC } \\
\text { nucle } \\
\text { i \# }\end{array}$ & size & $\begin{array}{l}\mathbb{0} \\
\mathbb{\pi} \\
\stackrel{\pi}{0} \\
\underset{\pi}{\pi}\end{array}$ \\
\hline 1. & $\begin{array}{l}0259 \\
6\end{array}$ & $\begin{array}{l}0.01 \\
8\end{array}$ & 21. & 116 & $\begin{array}{l}0.00 \\
1\end{array}$ & 41. & 19 & 0.0 & 61. & 6 & 0.0 \\
\hline 2. & $\begin{array}{l}0708 \\
4\end{array}$ & $\begin{array}{l}0.04 \\
8\end{array}$ & 22. & 42 & 0.0 & 42. & 23 & 0.0 & 62. & 6531 & $\begin{array}{l}0.04 \\
5\end{array}$ \\
\hline 3. & 6 & 0.0 & 23. & 12 & 0.0 & 43. & 142 & $\begin{array}{l}0.00 \\
1\end{array}$ & 63. & 84 & $\begin{array}{l}0.00 \\
1\end{array}$ \\
\hline 4. & 7 & 0.0 & 24. & 7 & 0.0 & 44. & 27 & 0.0 & 64. & 131 & $\begin{array}{l}0.00 \\
1\end{array}$ \\
\hline 5. & 48 & 0.0 & 25. & 28 & 0.0 & 45. & 6474 & $\begin{array}{l}0.04 \\
4\end{array}$ & 65. & 6904 & $\begin{array}{l}0.04 \\
7\end{array}$ \\
\hline 6. & 9197 & $\begin{array}{l}0.06 \\
3\end{array}$ & 26. & 54212 & $\begin{array}{l}0.37 \\
1\end{array}$ & 46. & 16 & 0.0 & 66. & 48 & 0.0 \\
\hline
\end{tabular}




\begin{tabular}{|c|c|c|c|c|c|c|c|c|c|c|c|}
\hline 7. & 227 & $\begin{array}{l}0.00 \\
2\end{array}$ & 27. & 43 & 0.0 & 47. & 37 & 0.0 & 67. & 177 & $\begin{array}{l}0.00 \\
1\end{array}$ \\
\hline 8. & $\begin{array}{l}1412 \\
8\end{array}$ & $\begin{array}{l}0.09 \\
7\end{array}$ & 28. & 83 & $\begin{array}{l}0.00 \\
1\end{array}$ & 48. & 7388 & $\begin{array}{l}0.05 \\
1\end{array}$ & 68. & 100 & $\begin{array}{l}0.00 \\
1\end{array}$ \\
\hline 9. & 185 & $\begin{array}{l}0.00 \\
1\end{array}$ & 29. & 3 & 0.0 & 49. & 9587 & $\begin{array}{l}0.06 \\
6\end{array}$ & 69. & 3 & 0.0 \\
\hline 10. & 27 & 0.0 & 30. & 6 & 0.0 & 50. & 19 & 0.0 & 70. & 3 & 0.0 \\
\hline 11. & 2 & 0.0 & 31. & 1136 & $\begin{array}{l}0.00 \\
8\end{array}$ & 51. & 916 & $\begin{array}{l}0.06 \\
6\end{array}$ & 71. & 111 & $\begin{array}{l}0.00 \\
1\end{array}$ \\
\hline 12. & 109 & $\begin{array}{l}0.00 \\
1\end{array}$ & 32. & 5 & 0.0 & 52. & 88 & $\begin{array}{l}0.00 \\
1\end{array}$ & 72. & 4 & 0.0 \\
\hline 13. & 22 & 0.0 & 33. & 11167 & $\begin{array}{l}0.07 \\
6\end{array}$ & 53. & 471 & $\begin{array}{l}0.00 \\
3\end{array}$ & 73. & 15 & 0.0 \\
\hline 14. & 37 & 0.0 & 34. & 39 & 0.0 & 54. & 176 & $\begin{array}{l}0.00 \\
1\end{array}$ & 74. & 4687 & $\begin{array}{l}0.03 \\
2\end{array}$ \\
\hline 15. & 17 & 0.0 & 35. & 81 & $\begin{array}{l}0.00 \\
1\end{array}$ & 55. & 14 & 0.0 & 75. & 16 & 0.0 \\
\hline 16. & 115 & $\begin{array}{l}0.00 \\
1\end{array}$ & 36. & 28 & 0.0 & 56. & 10 & 0.0 & 76. & 65 & 0.0 \\
\hline 17. & 6 & 0.0 & 37. & 44 & 0.0 & 57. & 34 & 0.0 & 77. & 17 & 0.0 \\
\hline 18. & 8 & 0.0 & 38. & 99 & $\begin{array}{l}0.00 \\
1\end{array}$ & 58. & 32 & 0.0 & 78. & 26 & 0.0 \\
\hline 19. & 1 & 0.0 & 39. & 613 & $\begin{array}{l}0.00 \\
4\end{array}$ & 59. & 14 & 0.0 & 79. & 1 & 0.0 \\
\hline 20 & 32 & 0.0 & 40 & 25 & 0.0 & 60. & 3 & 0.0 & 80. & 3 & 0.0 \\
\hline & & & & & & & & 0.0 & 81. & 1 & 0.0 \\
\hline
\end{tabular}

Table (4)Relative size (area) of each object w.r.t average

WBC area less than or equal threshold

\begin{tabular}{|l|l|l|}
\hline $\begin{array}{l}\text { WBC } \\
\text { nuclei } \\
\#\end{array}$ & size & average \\
\hline 1 & 2596 & 0.018 \\
\hline 2 & 7084 & 0.048 \\
\hline 3 & 9197 & 0.063 \\
\hline 4 & 14128 & $\mathbf{0 . 0 9 7}$ \\
\hline 5 & 54212 & 0.371 \\
\hline 6 & 11167 & $\mathbf{0 . 0 7 6}$ \\
\hline 7 & 6474 & $\mathbf{0 . 0 4 6}$ \\
\hline
\end{tabular}

\begin{tabular}{|l|l|l|}
\hline 8 & $\mathbf{7 3 8 8}$ & $\mathbf{0 . 0 4 4}$ \\
\hline 9 & $\mathbf{9 5 8 7}$ & $\mathbf{0 . 0 6 6}$ \\
\hline 10 & $\mathbf{6 5 3 1}$ & $\mathbf{0 . 0 4 5}$ \\
\hline 11 & $\mathbf{6 9 0 4}$ & $\mathbf{0 . 0 4 7}$ \\
\hline 12 & $\mathbf{4 6 8 7}$ & $\mathbf{0 . 0 3 2}$ \\
\hline
\end{tabular}




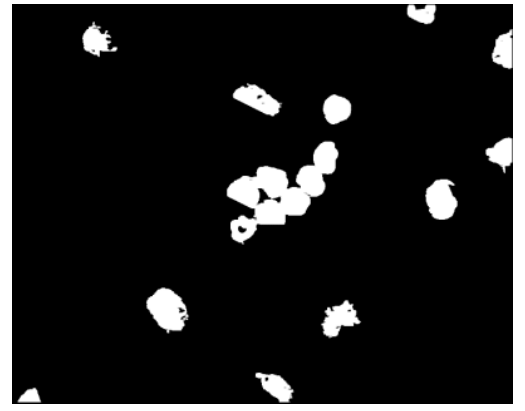

Fig (21): ALL-IDB1 Im001_1 image after segmentation

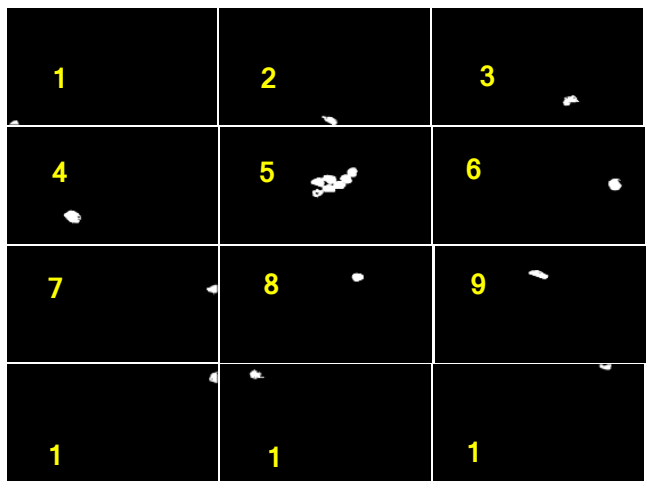

In our proposal ALLCD system the features extracted from nuclei including area, perimeter, and circularity. Table (5) is shown WBC nuclei size of connected objects, its perimeter and circularity

Table (5) WBC nuclei size area, perimeter and circularity

[1] WBC nuclei \# size perimeter circularity

[2] $1 . \quad 2596 \quad 98 \quad 3.397$

[3] $2 . \quad 7084 \quad 274 \quad 1.186$

[4] 3. $\quad 9197 \quad 376 \quad 0.817$

[5] $4 . \quad 14128 \quad 416 \quad 1.026$

[6] $5 . \quad 54212 \quad 1246 \quad 0.439$

[7] $6 . \quad 11167 \quad 290 \quad 1.669$

[8] $7 . \quad 6474 \quad 256 \quad 1.241$

$\begin{array}{lllll}{[9]} & 8 . & 7388 & 204 & 2.231\end{array}$

[10] $9 . \quad 9587 \quad 296 \quad 1.375$

[11] $10 . \quad 6531 \quad 306 \quad 0.876$

[12] 11. $\quad 6904 \quad 354 \quad 0.692$

$\begin{array}{llll}12 . & 4687 & 184 & 1.74\end{array}$

Fig (22): Segmented WBC nuclei Label number

Table (6): The distance between WBC nuclei

\begin{tabular}{|c|c|c|c|c|c|c|c|c|c|c|c|c|}
\hline $\begin{array}{l}\text { WBC } \\
\text { \# }\end{array}$ & 01 & 02 & 03 & 04 & 05 & 06 & 07 & 08 & 09 & 10 & 11 & 12 \\
\hline 01 & 0000 & 0771 & 1110 & 0561 & 1316 & 1575 & 1834 & 1434 & 1270 & 2039 & 1268 & 1893 \\
\hline 02 & 0771 & 0000 & & & 0827 & 0917 & 1172 & 0985 & 0990 & 1456 & 1327 & 1392 \\
\hline 03 & 1110 & 0389 & & 0436 & 0546 & 0533 & 0784 & 0704 & 0816 & 1083 & 1287 & 1053 \\
\hline 04 & 0561 & 0436 & 0 & 0626 & 0756 & 1029 & 1284 & 0879 & 0754 & 1478 & 0940 & 1336 \\
\hline 05 & 1316 & 0827 & 0626 & 00 & 0000 & 0414 & 0610 & 0161 & 0345 & 0728 & 0906 & 0587 \\
\hline 06 & 1575 & 0917 & 0546 & 0000 & 0414 & 0000 & 0260 & 0477 & 0751 & 0560 & 1316 & 0597 \\
\hline 07 & 1834 & 1172 & & 0756 & 0610 & 0260 & 0000 & 0614 & 0912 & 0370 & 1472 & 0520 \\
\hline 08 & 1434 & 0985 & & ט & 0161 & 0477 & 0614 & 0000 & 0299 & 0644 & 0859 & 0459 \\
\hline 09 & 1270 & 0990 & $\begin{array}{l}0704 \\
0816\end{array}$ & 1029 & 0345 & 0751 & 0912 & 0299 & 0000 & 0920 & 0566 & 0692 \\
\hline 10 & 2039 & 1456 & 0816 & & 0728 & 0560 & 0370 & 0644 & 0920 & 0000 & 1426 & 0276 \\
\hline 11 & 1268 & 1327 & 1083 & .284 & 0906 & 1316 & 1472 & 0859 & 0566 & 1426 & 0000 & 1164 \\
\hline 12 & 1893 & 1392 & 1287 & 0879 & 0587 & 0597 & 0520 & 0459 & 0692 & 0276 & 1164 & 0000 \\
\hline
\end{tabular}

The WBC classification result from applying KNN algorithm with respect to Euclidean's distances among nuclei's according to table (6). And $\mathrm{k}=1$ is shown in table (7).

Table (7):classification results

\begin{tabular}{|l|l|l|l|l|l|l|}
\hline $\begin{array}{l}\text { WBC } \\
\#\end{array}$ & Area & $\begin{array}{l}\text { perim } \\
\text { eter }\end{array}$ & $\begin{array}{l}\text { Circula } \\
\text { rity }\end{array}$ & $\begin{array}{l}\text { Dista } \\
\text { nce }\end{array}$ & $\begin{array}{l}\text { Neare } \\
\text { st } \\
\text { Neigh } \\
\text { bor }\end{array}$ & $\begin{array}{l}\text { Normal } \\
\text { or Blast }\end{array}$ \\
\hline 1. & $\mathbf{2 5 9 6}$ & 98 & 3.397 & $\mathbf{5 6 1}$ & $\mathbf{4}$ & Normal \\
\hline 2. & $\mathbf{7 0 8 4}$ & 274 & 1.186 & $\mathbf{3 8 9}$ & $\mathbf{3}$ & Blast \\
\hline 3. & $\mathbf{9 1 9 7}$ & 376 & 0.817 & $\mathbf{3 8 9}$ & $\mathbf{2}$ & Blast \\
\hline 4. & $\mathbf{1 4 1 2 8}$ & 416 & 1.026 & $\mathbf{4 3 6}$ & $\mathbf{2}$ & Normal \\
\hline
\end{tabular}

\begin{tabular}{|l|l|l|l|l|l|l|}
\hline 5. & $\mathbf{5 4 2 1 2}$ & 1246 & 0.439 & $\mathbf{3 4 5}$ & $\mathbf{9}$ & Blast \\
\hline 6. & $\mathbf{1 1 1 6 7}$ & 290 & 1.669 & $\mathbf{2 6 0}$ & $\mathbf{7}$ & Normal \\
\hline 7. & $\mathbf{6 4 7 4}$ & 256 & 1.241 & $\mathbf{2 6 0}$ & $\mathbf{6}$ & Normal \\
\hline 8. & $\mathbf{7 3 8 8}$ & 204 & 2.231 & $\mathbf{1 6 1}$ & $\mathbf{5}$ & Blast \\
\hline 9. & $\mathbf{9 5 8 7}$ & 296 & 1.375 & $\mathbf{2 9 9}$ & $\mathbf{8}$ & Blast \\
\hline 10. & $\mathbf{6 5 3 1}$ & 306 & 0.876 & $\mathbf{2 7 6}$ & $\mathbf{1 2}$ & Blast \\
\hline 11. & $\mathbf{6 9 0 4}$ & 354 & 0.692 & $\mathbf{5 6 6}$ & $\mathbf{9}$ & Blast \\
\hline 12. & $\mathbf{4 6 8 7}$ & 184 & 1.74 & $\mathbf{2 7 6}$ & $\mathbf{1 0}$ & Blast \\
\hline
\end{tabular}




\section{CONCLUSIONS}

1. The best accuracy of segmentation can be reached by using minimum nucleus segment threshold value.

2. This proposal system involves automated detection acute lymphocyte leukemia using microscopic blood sample images was obtained from ALL-IDB1.

3. The proposal system will be built by using features in microscopic images by examining area, perimeter and circularity as a KNN-classifier input.

4. The system should be efficient, reliable, less processing time, smaller error, high accuracy, cheaper cost and must be robust towards varieties that exist in individual.

5. The efficiency is increased by using the automated techniques.

6. Information extracted from microscopic images of blood samples can benefit people by predicting, solving and treating blood diseases immediately for a particular patient.

\section{REFERENCES}

[1] Minal and et at, DETECTION OF ACUTE LEUKEMIA USING WHITE BLOOD CELLS SEGMENTATION BASED ON BLOOD SAMPLES, interational Journal of Electronics and Communication Engineering and Technology Communication,p148,june,2013

[2] HAYAN TAREQ ABDUL WAHHAB, "CLASSIFICATION OF ACUTE LEUKEMIA USING IMAGEPROCESSING AND MACHINE LEARNING TECHNIQUES", PHD thesis, FACULTY OF COMPUTER SCIENCE and INFORMATION TECHNOLOGY UNIVERSITY OF MALAYA, KUALA LUMPUR, 2015.

[3] Sabino, D., Costa, L. d. F., Martins, S., Calado, R., and Zago, M.Automatic leukemia diagnosis. Acta Microscopica, 12(1), 1-6,2003.

[4] N., Ritter, J., Cooper, "Segmentation and Border Identification of Cells in Images of Peripheral Blood Smear Slides", 30th Australasian Computer Science Conference, Conference in Research and Practice in Information Technology, Vol. 62, pp. 161-169, 2007.

[5] Mehnert and P. Jackway, "An improved seeded region growing algorithm," Pattern Recognition Letters, vol. 18, no. 10, pp. 1065-1071, 1997.

[6] T. Mouroutis, S. J. Roberts, and A. A. Bharath, "Robust cell nuclei segmentation using statistical modelling," Bioimaging, vol. 6, no. 2, pp. 79-91, 1998. 